\title{
The Bound of the Hankel Determinant of the Third Kind for Starlike Functions
}

\section{Oh Sang Kwon ${ }^{1} \cdot$ Adam Lecko $^{2}$ (D) Young Jae Sim ${ }^{1}$}

Received: 10 November 2017 / Revised: 28 May 2018 / Published online: 13 October 2018

(c) The Author(s) 2018

\section{Abstract}

In the present paper, the estimate of the third Hankel determinant

$$
H_{3,1}(f)=\left|\begin{array}{lll}
a_{1} & a_{2} & a_{3} \\
a_{2} & a_{3} & a_{4} \\
a_{3} & a_{4} & a_{5}
\end{array}\right|
$$

for the class of starlike functions, i.e., for the class of analytic functions $f$ standardly normalized such that $\operatorname{Re}\left(z f^{\prime}(z) / f(z)\right)>0, z \in \mathbb{D}:=\{z \in \mathbb{C}:|z|<1\}$, is improved.

Keywords Univalent functions - Starlike functions · Carathéodory functions · Hankel determinant $\cdot$ Fourth coefficient

Mathematics Subject Classification Primary 30C45

\section{Introduction}

Let $\mathcal{H}$ be a class of analytic functions in $\mathbb{D}:=\{z \in \mathbb{C}:|z|<1\}$ and let $\mathcal{A}$ be its subclass normalized by $f(0):=0, f^{\prime}(0):=1$, i.e., of the form

Communicated by Saminathan Ponnusamy.

$\triangle$ Adam Lecko

alecko@matman.uwm.edu.pl

Oh Sang Kwon

oskwon@ks.ac.kr

Young Jae Sim

yjsim@ks.ac.kr

1 Department of Mathematics, Kyungsung University, Busan 48434, Korea

2 Department of Complex Analysis, Faculty of Mathematics and Computer Science, University of Warmia and Mazury in Olsztyn, Ul. Słoneczna 54, 10-710 Olsztyn, Poland 


$$
f(z)=\sum_{n=1}^{\infty} a_{n} z^{n}, \quad a_{1}:=1, z \in \mathbb{D} .
$$

Let $\mathcal{S}^{*}$ denote the class of starlike functions, namely, the subclass of $\mathcal{A}$ consisting of functions $f$ such that

$$
\operatorname{Re} \frac{z f^{\prime}(z)}{f(z)}>0, \quad z \in \mathbb{D} .
$$

Given $q, n \in \mathbb{N}$, the Hankel determinants $H_{q, n}(f)$ of Taylor's coefficients of functions $f \in \mathcal{A}$ of the form (1.1) are defined as

$$
H_{q, n}(f):=\left|\begin{array}{cccc}
a_{n} & a_{n+1} & \cdots & a_{n+q-1} \\
a_{n+1} & a_{n+2} & \cdots & a_{n+q} \\
\vdots & \vdots & \vdots & \vdots \\
a_{n+q-1} & a_{n+q} & \cdots & a_{n+2(q-1)}
\end{array}\right| .
$$

Particularly, the third Hankel determinant $H_{3,1}(f)$ is given by

$$
H_{3,1}(f):=\left|\begin{array}{lll}
a_{1} & a_{2} & a_{3} \\
a_{2} & a_{3} & a_{4} \\
a_{3} & a_{4} & a_{5}
\end{array}\right|=a_{3}\left(a_{2} a_{4}-a_{3}^{2}\right)-a_{4}\left(a_{4}-a_{2} a_{3}\right)+a_{5}\left(a_{3}-a_{2}^{2}\right)
$$

To find the growth of the Hankel determinant $H_{q, n}(f)$ dependent on $q$ and $n$ for the whole class $\mathcal{S} \subset \mathcal{A}$ of univalent functions as well as for its subclasses is an interesting problem to study. For the class $\mathcal{S}$ some important result was shown by Pommerenke [13]. For fixed $q$ and $n$ the growth problem can be reduced to an estimate of the Hankel determinant for the selected subclasses of $\mathcal{A}$. Recently many authors examined the Hankel determinant $H_{2,2}(f)=a_{2} a_{4}-a_{3}^{2}$ of order 2 (see, e.g., [3,4,6,8,12]). Note also that $H_{2,1}(f)=a_{3}-a_{2}^{2}$. Thus the Hankel determinant $H_{2,1}(f)$ reduces to the well-known coefficient functional which for $\mathcal{S}$ was estimated in 1916 by Bieberbach (see, e.g., [5, Vol. I, p. 35]).

The problem to find the upper bound of the Hankel determinant $H_{3,1}(f)$ of order 3 is more sophisticated if we expect to get sharp result. From (1.3) by using the triangle inequality we get at once the following inequality

$$
\left|H_{3,1}(f)\right| \leq\left|a_{3}\right|\left|H_{2,2}(f)\right|+\left|a_{4}\right|\left|a_{4}-a_{2} a_{3}\right|+\left|a_{5}\right|\left|H_{2,1}(f)\right| .
$$

This simple observation allowed to estimate of $\left|H_{3,1}(f)\right|$ for compact subclasses $\mathcal{F}$ of $\mathcal{A}$ by various authors (see, e.g., [2,15-18]). However, these results are far from sharpness. If case when a given subclass $\mathcal{F}$ of $\mathcal{A}$ has a representation with using the Carathéodory class $\mathcal{P}$, i.e., the class of functions $p \in \mathcal{H}$ of the form

$$
p(z)=1+\sum_{n=1}^{\infty} c_{n} z^{n}, \quad z \in \mathbb{D}
$$


having a positive real part in $\mathbb{D}$, the coefficients of functions in $\mathcal{F}$ have a suitable representation expressed by the coefficients of functions in $\mathcal{P}$. Therefore to get the upper bound of each term in (1.4) cited authors based their computing on the wellknown formulas on coefficient $c_{2}$ (e.g., $\left[14\right.$, p. 166]) and on the formula $c_{3}$ due to Libera and Zlotkiewicz [9].

In order to improve the bound of $\left|H_{3,1}(f)\right|$ we have to use directly formula (1.3), where we need to apply a formula for $c_{4}$, similar to the formulas (2.1) and (2.2). In a recent paper [7] the authors found such a formula for $c_{4}$. According to the authors' knowledge, formulas for the coefficients $c_{n}$ for $n \geq 5$ analogous to the formulas (2.1) and (2.2) are not known.

Basing on the formulas for $c_{2}, c_{3}$ and $c_{4}$, we improve the known estimate of the Hankel determinant $H_{3,1}(f)$ in the class $\mathcal{S}^{*}$ of starlike functions. We show that $\left|H_{3,1}(f)\right| \leq 8 / 9$. Estimating each term of the right hand of (1.4) Babalola [1] showed that $\left|H_{3,1}(f)\right| \leq 16$. In [19] Zaprawa by a suitable grouping and using Lemma 1 due to Livingston [11] proved that $\left|H_{3,1}(f)\right| \leq 1$.

\section{Main Result}

The basis for proof of the main result is the following lemma. It contains the wellknown formula for $c_{2}$ (e.g., [14, p. 166]), the formula for $c_{3}$ due to Libera and Zlotkiewicz $[9,10]$ and the formula for $c_{4}$ found by the authors [7].

Lemma 2.1 If $p \in \mathcal{P}$ is of the form (1.5) with $c_{1} \geq 0$, then

$$
\begin{aligned}
& 2 c_{2}=c_{1}^{2}+\left(4-c_{1}^{2}\right) \zeta \\
& 4 c_{3}=c_{1}^{3}+\left(4-c_{1}^{2}\right) c_{1} \zeta(2-\zeta)+2\left(4-c_{1}^{2}\right)\left(1-|\zeta|^{2}\right) \eta
\end{aligned}
$$

and

$$
\begin{aligned}
8 c_{4} & =c_{1}^{4}+\left(4-c_{1}^{2}\right) \zeta\left[c_{1}^{2}\left(\zeta^{2}-3 \zeta+3\right)+4 \zeta\right] \\
& -4\left(4-c_{1}^{2}\right)\left(1-|\zeta|^{2}\right)\left[c_{1}(\zeta-1) \eta+\bar{\zeta} \eta^{2}-\left(1-|\eta|^{2}\right) \xi\right]
\end{aligned}
$$

for some $\zeta, \eta, \xi \in \overline{\mathbb{D}}:=\{z \in \mathbb{C}:|z| \leq 1\}$.

Now, we will estimate the third-order Hankel determinant $H_{3,1}(f)$ for $f \in \mathcal{S}^{*}$. To this end, the following propositions are required.

Proposition 2.2 Let $\Theta:[0,3] \times[0,1] \rightarrow \mathbb{R}$ be a function defined by

$$
\Theta(t, x):=96 \theta_{1}(x)-8 \theta_{2}(x) t+3 \theta_{3}(x) t^{2},
$$

where for $x \in[0,1]$,

$$
\begin{aligned}
& \theta_{1}(x):=2+8 x-x^{2}-6 x^{3} \\
& \theta_{2}(x):=16+67 x-34 x^{2}-53 x^{3}+2 x^{4}
\end{aligned}
$$


and

$$
\theta_{3}(x):=12+19 x-21 x^{2}-17 x^{3}+3 x^{4}
$$

Then $\Theta(t, x)>0$ for $0 \leq t \leq 3$ and $0 \leq x \leq 1$.

Proof At first, note that the polynomial $\theta_{3}$ has a unique zero $x=: x_{1} \approx 0.9314$ in $(0,1)$. Since $x_{1} \in(0.92,0.95)$ and for $x \in(0.92,0.95)$,

$$
\begin{aligned}
& \theta_{2}(x)>16+67 \cdot(0.92)-34 \cdot(0.95)^{2}-53 \cdot(0.95)^{3}+2 \cdot(0.92)^{4} \\
& \quad=2.94691092>0
\end{aligned}
$$

it follows that

$$
\frac{\partial}{\partial t} \Theta\left(t, x_{1}\right)=-8 \theta_{2}\left(x_{1}\right) \neq 0
$$

For $x \neq x_{1},(\partial / \partial t) \Theta(t, x)=0$ occurs at

$$
t=\frac{4 \theta_{2}(x)}{3 \theta_{3}(x)}=: t_{0}(x) .
$$

We have

$$
\left.\frac{\partial}{\partial x} \Theta(t, x)\right|_{t=t_{0}(x)}=\frac{16 \theta_{4}(x)}{9 \theta_{3}^{2}(x)},
$$

where

$$
\begin{aligned}
\theta_{4}(x):= & 54 \theta_{1}^{\prime}(x) \theta_{3}^{2}(x)-6 \theta_{2}^{\prime}(x) \theta_{2}(x) \theta_{3}(x)+3 \theta_{3}^{\prime}(x) \theta_{2}^{2}(x) \\
= & -3\left(128+31896 x-18709 x^{2}-133828 x^{3}-3737 x^{4}+198602 x^{5}\right. \\
& +74185 x^{6}-91136 x^{7}-54071 x^{8}-2774 x^{9}+668 x^{10} \\
& \left.+48 x^{11}\right), \quad x \in(0,1) .
\end{aligned}
$$

The polynomial $\theta_{4}$ has exactly two zeros in $(0,1)$, namely, $x=: x_{2} \approx 0.533701$ and $x=: x_{3} \approx 0.811327$. We will now show that

$$
t_{0}(x)>3, \quad x \in[0.5,0.9]
$$

Since $x_{1}>0.9$, so $\theta_{3}(x)>0$, for $x \in[0.5,0.9]$ and the inequality (2.5) is equivalent to

$$
4 \theta_{2}(x)-9 \theta_{3}(x)>0, \quad x \in[0.5,0.9]
$$


The above one can be equivalently written as

$$
19 x^{4}+59 x^{3}-53 x^{2}-97 x+44<0, \quad x \in[0.5,0.9] .
$$

As the polynomial on the left hand of the above inequality has a unique zero $x \approx$ 0.40928 in $[0,1]$, the above inequality is true, so is the inequality (2.5). Thus the function $\Theta$ has no critical point in $(0,3) \times(0,1)$. Hence it is sufficient to show that $\Theta>0$ on the boundary of $[0,3] \times[0,1]$. We can easily check that the following inequalities hold:

$$
\begin{aligned}
& \Theta(t, 0)=4\left(48-32 t+9 t^{2}\right) \geq \frac{704}{9}, \quad t \in[0,3], \\
& \Theta(t, 1)=4\left(72+4 t-3 t^{2}\right) \geq 228, \quad t \in[0,3], \\
& \Theta(0, x)=96\left(2+8 x-x^{2}-6 x^{3}\right) \geq 192, \quad x \in[0,1],
\end{aligned}
$$

and

$$
\begin{aligned}
\Theta(3, x) & =3\left(44-109 x+51 x^{2}+79 x^{3}+11 x^{4}\right) \\
& =3\left(44(1-x)^{3}+x\left(23-81 x+123 x^{2}\right)+11 x^{4}\right) \\
& \geq 3\left(44(1-x)^{3}+\frac{1585}{164} x+11 x^{4}\right)>0, \quad x \in[0,1] .
\end{aligned}
$$

Thus the proof of the proposition is completed.

Proposition 2.3 Let $\Psi:[1,4] \times[0,1] \rightarrow \mathbb{R}$ be a function defined by

$$
\Psi(t, x):=16 \psi_{1}(x)+8 \psi_{2}(x) t+3 \psi_{3}(x) t^{2},
$$

where for $x \in[0,1]$,

$$
\begin{aligned}
& \psi_{1}(x):=-2+27 x+21 x^{2}-37 x^{3}+x^{4}, \\
& \psi_{2}(x):=10-12 x-9 x^{2}+20 x^{3}+x^{4}
\end{aligned}
$$

and

$$
\psi_{3}(x):=x\left(3-5 x-x^{2}-x^{3}\right)
$$

Then $\Psi(t, x)>0$ for $1 \leq t \leq 4$ and $0 \leq x \leq 1$.

Proof At first, note that the function $\psi_{3}$ has a unique zero $x=: x_{1} \approx 0.51839$ in $(0,1)$. Since $x_{1} \in(0.5,0.6)$ and for $x \in(0.5,0.6)$,

$$
\psi_{2}(x)>10-12 \cdot(0.6)-9 \cdot(0.6)^{2}+20 \cdot(0.5)^{3}+(0.5)^{4}=2.1225>0,
$$

it follows that

$$
\frac{\partial}{\partial t} \Psi\left(t, x_{1}\right)=8 \psi_{2}\left(x_{1}\right) \neq 0
$$


For $x \neq x_{1},(\partial / \partial t) \Psi(t, x)=0$ occurs at

$$
t=\frac{-4 \psi_{2}(x)}{3 \psi_{3}(x)}=: t_{0}(x) .
$$

We have

$$
\left.\frac{\partial}{\partial x} \Psi(t, x)\right|_{t=t_{0}(x)}=\frac{16 \psi_{4}(x)}{3 \psi_{3}^{2}(x)}
$$

where

$$
\begin{aligned}
\psi_{4}(x): & =3 \psi_{1}^{\prime}(x) \psi_{3}^{2}(x)-2 \psi_{2}^{\prime}(x) \psi_{2}(x) \psi_{3}(x)+\psi_{3}^{\prime}(x) \psi_{2}^{2}(x) \\
= & 300-1000 x+1737 x^{2}-4912 x^{3}+2009 x^{4}+13706 x^{5}-17777 x^{6} \\
& +6596 x^{7}-1541 x^{8}+546 x^{9}-184 x^{10}+16 x^{11}, \quad x \in(0,1) .
\end{aligned}
$$

The polynomial $\psi_{4}$ has a unique zero $x=: x_{2} \approx 0.388025$ in $(0,1)$. Since $x_{1}>0.5$, so $\psi_{3}(x)>0$, for $x \in(0,0.5)$. Additionally, since $\psi_{2}$ has no zero in $(0,1)$, the inequality (2.7) is true on $[0,1]$. Thus $t_{0}(x)<0$ for $x \in(0,0.5)$ and in consequence, the function $\Psi$ has no critical point in $(1,4) \times(0,1)$. Hence it is sufficient to show that $\Psi>0$ on the boundary of $[1,4] \times[0,1]$. We can easily check that the following inequalities hold:

$$
\begin{aligned}
& \Psi(t, 0)=-32+80 t \geq 48, \quad t \in[1,4] \\
& \Psi(t, 1)=160+80 t-12 t^{2} \geq 228, \quad t \in[1,4], \\
& \Psi(4, x)=96\left(3+2 x-2 x^{2}\right) \geq 288, \quad x \in[0,1],
\end{aligned}
$$

and

$$
\begin{aligned}
\Psi(1, x) & =3\left(16+115 x+83 x^{2}-145 x^{3}+7 x^{4}\right) \\
& =3\left(16+53 x^{2}+7 x^{4}+115 x\left(1-x^{2}\right)+30 x^{2}(1-x)\right) \\
& \geq 48, \quad x \in[0,1] .
\end{aligned}
$$

Thus the proof of the proposition is completed.

Proposition 2.4 Let $\Phi:[3,4] \times[0,1] \rightarrow \mathbb{R}$ be a function defined by

$$
\Phi(t, x):=48 \phi_{1}(x)+8 \phi_{2}(x) t-3 \phi_{3}(x) t^{2},
$$

where for $x \in[0,1]$,

$$
\begin{aligned}
& \phi_{1}(x):=1+7 x+x^{2}-3 x^{3} \\
& \phi_{2}(x):=5-19 x+10 x^{2}+5 x^{3}+x^{4}
\end{aligned}
$$

and

$$
\phi_{3}(x):=x\left(-3+5 x+x^{2}+x^{3}\right)
$$

Then $\Phi(t, x)>0$ for $3 \leq t \leq 4$ and $0 \leq x \leq 1$. 
Proof Since $\phi_{3}=-\psi_{3}$, by the part of proof of Proposition 2.3, we at once have

$$
\frac{\partial}{\partial t} \Phi\left(t, x_{1}\right)=8 \phi_{2}\left(x_{1}\right) \neq 0
$$

For $x \neq x_{1},(\partial / \partial t) \Phi(t, x)=0$ occurs at

$$
t=\frac{4 \phi_{2}(x)}{3 \phi_{3}(x)}=: t_{0}(x) .
$$

We have

$$
\left.\frac{\partial}{\partial x} \Phi(t, x)\right|_{t=t_{0}(x)}=\frac{16 \phi_{4}(x)}{3 \phi_{3}^{2}(x)},
$$

where

$$
\begin{aligned}
\phi_{4}(x):= & 9 \phi_{1}^{\prime}(x) \phi_{3}^{2}(x)+2 \phi_{2}^{\prime}(x) \phi_{2}(x) \phi_{3}(x)-\phi_{3}^{\prime}(x) \phi_{2}^{2}(x) \\
= & 75-250 x+59 x^{2}+532 x^{3}-893 x^{4}+558 x^{5}-269 x^{6}+844 x^{7} \\
& -366 x^{8}+16 x^{9}-46 x^{10}+4 x^{11}, \quad x \in(0,1) .
\end{aligned}
$$

The polynomial $\phi_{4}$ has exactly two zeros in $(0,1)$, namely $x=: x_{2} \approx 0.414034$ and $x=: x_{3} \approx 0.663886$. We have $t_{0}\left(x_{2}\right) \approx 3.59845$ and $t_{0}\left(x_{3}\right)=-2.95522$. Therefore the function $\Phi$ has a unique critical point $\left(t_{0}\left(x_{2}\right), x_{2}\right)$ in $(3,4) \times(0,1)$. For $(t, x) \in[3.58,3.61] \times[0.39,0.43]$ by simple computing, we show that $\Phi(t, x)>0$. Thus, particularly $\Phi\left(t_{0}\left(x_{2}\right), x_{2}\right)>0$. Therefore it is sufficient to show that $\Phi>0$ on the boundary of $[3,4] \times[0,1]$. We can easily check that the following inequalities hold:

$$
\begin{aligned}
& \Phi(t, 0)=8(6+5 t) \geq 168, \quad t \in[3,4] \\
& \Phi(t, 1)=4\left(72+4 t-3 t^{2}\right) \geq 160, \quad t \in[3,4] \\
& \Phi(4, x)=16\left(13-8 x+8 x^{2}-2 x^{3}-x^{4}\right) \geq 16\left(2+8 x^{2}\right) \geq 32, \quad x \in[0,1],
\end{aligned}
$$

and

$\Phi(3, x)=3\left(56-13 x+51 x^{2}-17 x^{3}-x^{4}\right) \geq 3\left(25+51 x^{2}\right) \geq 75, \quad x \in[0,1]$.

Thus the proof of the proposition is completed.

Finally, we estimate now the third-order Hankel determinant $H_{3,1}(f)$ for $f \in \mathcal{S}^{*}$.

Theorem 2.5 If $f \in \mathcal{S}^{*}$ is the form (1.1), then

$$
\left|H_{3,1}(f)\right| \leq \frac{8}{9} .
$$


Proof Let $f \in \mathcal{S}^{*}$ be of the form (1.1). Then by (1.2) we have

$$
z f^{\prime}(z)=f(z) p(z), \quad z \in \mathbb{D}
$$

for some function $p \in \mathcal{P}$ of the form (1.5). Since the class $\mathcal{P}$ is invariant under the rotations, we may assume that $c:=c_{1} \in[0,2]$ (e.g., [5, Vol. I, p. 80, Theorem 3]). Putting the series (1.1) and (1.5) into (2.10) and by equating the coefficients we get

$$
a_{2}=c, \quad a_{3}=\frac{1}{2}\left(c^{2}+c_{2}\right), \quad a_{4}=\frac{1}{6}\left(c^{3}+3 c c_{2}+2 c_{3}\right)
$$

and

$$
a_{5}=\frac{1}{24}\left(c^{4}+6 c^{2} c_{2}+8 c c_{3}+3 c_{2}^{2}+6 c_{4}\right) .
$$

Hence

$$
\begin{aligned}
H_{3,1}(f)= & -a_{3}^{3}+2 a_{2} a_{3} a_{4}-a_{4}^{2}-a_{2}^{2} a_{5}+a_{3} a_{5} \\
= & \frac{1}{144}\left(-c^{6}+3 c^{4} c_{2}-9 c_{2}^{3}+8 c^{3} c_{3}+24 c c_{2} c_{3}-16 c_{3}^{2}\right. \\
& \left.+18 c_{2} c_{4}-9 c^{2} c_{2}^{2}-18 c^{2} c_{4}\right) .
\end{aligned}
$$

Now using the equalities (2.1)-(2.3), by straightforward algebraic computation we have

$$
H_{3,1}(f)=\frac{1}{1152}\left(c^{2}-4\right)\left[\gamma_{1}(c, \zeta)+\gamma_{2}(c, \zeta) \eta+\gamma_{3}(c, \zeta) \eta^{2}+\Gamma(c, \zeta, \eta) \xi\right],
$$

where for $\zeta, \eta, \xi \in \overline{\mathbb{D}}$,

$$
\begin{aligned}
& \gamma_{1}(c, \zeta):=c^{2} \zeta\left[-3 c^{2}+\left(44-5 c^{2}\right) \zeta+\left(40-c^{2}\right) \zeta^{2}\right]-c^{2}\left(4-c^{2}\right) \zeta^{4}, \\
& \gamma_{2}(c, \zeta):=-4 c\left(1-|\zeta|^{2}\right)\left[3 c^{2}+4\left(5+c^{2}\right) \zeta-\left(4-c^{2}\right) \zeta^{2}\right] \\
& \gamma_{3}(c, \zeta):=32\left(4-c^{2}\right)-28|\zeta|^{2}\left(4-c^{2}\right)-36 c^{2} \bar{\zeta}\left(1-|\zeta|^{2}\right)-4\left(4-c^{2}\right)|\zeta|^{4},
\end{aligned}
$$

and

$$
\Gamma(c, \zeta, \eta):=36\left[c^{2}+\left(c^{2}-4\right) \zeta\right]\left(1-|\zeta|^{2}\right)\left(1-|\eta|^{2}\right)
$$


Setting $x:=|\zeta| \in[0,1], y:=|\eta| \in[0,1]$ and taking into account that $|\xi| \leq 1$, from (2.11) we get

$$
\begin{aligned}
& \left|H_{3,1}(f)\right| \\
& \quad \leq \frac{1}{1152}\left(4-c^{2}\right)\left[\left|\gamma_{1}(c, \zeta)\right|+\left|\gamma_{2}(c, \zeta)\right||\eta|+\left|\gamma_{3}(c, \zeta)\right||\eta|^{2}+|\Gamma(c, \zeta, \eta)|\right] \\
& \quad \leq \frac{1}{1152}\left(4-c^{2}\right) F(c, x, y),
\end{aligned}
$$

where

$$
F(c, x, y):=f_{1}(c, x)+f_{2}(c, x) y+f_{3}(c, x) y^{2}+f_{4}(c, x)\left(1-y^{2}\right),
$$

with

$$
\begin{aligned}
& f_{1}(c, x):=c^{2} x\left[3 c^{2}+\left(44-5 c^{2}\right) x+\left(40-c^{2}\right) x^{2}\right]+c^{2}\left(4-c^{2}\right) x^{4}, \\
& f_{2}(c, x):=4 c\left(1-x^{2}\right)\left[3 c^{2}+4\left(5+c^{2}\right) x+\left(4-c^{2}\right) x^{2}\right], \\
& f_{3}(c, x):=32\left(4-c^{2}\right)+28 x^{2}\left(4-c^{2}\right)+36 c^{2} x\left(1-x^{2}\right)+4\left(4-c^{2}\right) x^{4}
\end{aligned}
$$

and

$$
f_{4}(c, x):=36\left[c^{2}+\left(4-c^{2}\right) x\right]\left(1-x^{2}\right)
$$

Now, we will show that

$$
\left(4-c^{2}\right) F(c, x, y) \leq 1024
$$

for $c \in[0,2], x \in[0,1]$ and $y \in[0,1]$.

I. Assume first that $c \in[1,2]$. Then by (2.13) we have

$$
\begin{aligned}
& F(c, x, y) \\
& \quad \leq f_{1}(c, x)+c f_{2}(c, x) y+f_{3}(c, x) y^{2}+f_{4}(c, x)\left(1-y^{2}\right) \\
& \quad=f_{1}(c, x)+f_{4}(c, x)+c f_{2}(c, x) y+\left(f_{3}(c, x)-f_{4}(c, x)\right) y^{2} \\
& \quad=: F_{1}(c, x, y) .
\end{aligned}
$$

(a) Consider the case $f_{3}(c, x) \geq f_{4}(c, x)$ in $[1,2] \times[0,1]$. Let

$$
\Omega_{1}:=\left\{(c, x) \in[1,2] \times[0,1]: f_{3}(c, x) \geq f_{4}(c, x)\right\} .
$$

By (2.15) we get

$$
\begin{aligned}
F_{1}(c, x, y) & \leq F_{1}(c, x, 1) \\
& =f_{1}(c, x)+c f_{2}(c, x)+f_{3}(c, x), \quad(c, x) \in \Omega_{1}, y \in[0,1] .
\end{aligned}
$$


Set $t:=4-c^{2}$. Clearly, $t \in[0,3]$. Define

$$
\tilde{F}_{1}(t, x):=t F_{1}(\sqrt{4-t}, x, 1), \quad(\sqrt{4-t}, x) \in \Omega_{1}
$$

A simple computing yields

$$
\begin{aligned}
\tilde{F}_{1}(t, x)= & t\left\{(4-t) x\left[12-3 t+(24+5 t) x+(36+t) x^{2}\right]\right. \\
& +t(4-t) x^{4}+32 t+28 t x^{2}+36(4-t) x\left(1-x^{2}\right)+4 t x^{4} \\
& \left.+4(4-t)\left(1-x^{2}\right)\left[12-3 t+4(9-t) x+t x^{2}\right]\right\} \\
= & 96\left(2+8 x-x^{2}-6 x^{3}\right) t-4\left(16+67 x-34 x^{2}-53 x^{3}+2 x^{4}\right) t^{2} \\
& +\left(12+19 x-21 x^{2}-17 x^{3}+3 x^{4}\right) t^{3}, \quad(\sqrt{4-t}, x) \in \Omega_{1} .
\end{aligned}
$$

Hence and by Proposition 2.2 we have

$$
\frac{\partial}{\partial t} \tilde{F}_{1}(t, x)=\Theta(t, x)>0, \quad(\sqrt{4-t}, x) \in \Omega_{1},
$$

where the function $\Theta$ is defined by (2.4). Thus the function $[0,3] \ni t \mapsto \tilde{F}_{1}(t, \cdot)$ is increasing, and therefore we have

$$
\tilde{F}_{1}(t, x) \leq \tilde{F}_{1}(3, x)=9\left(36+45 x+41 x^{2}-31 x^{3}+x^{4}\right)<1024, \quad x \in[0,1] .
$$

Indeed, the last inequality is true since, as easy to verify the inequality

$$
-700+405 x+369 x^{2}-279 x^{3}+9 x^{4}<0, \quad x \in[0,1]
$$

holds. Thus the inequality (2.16) confirms the inequality (2.14).

(b) Consider the case $f_{3}(c, x)<f_{4}(c, x)$ in $[1,2] \times[0,1]$. Let

$$
\Omega_{2}:=\left\{(c, x) \in[1,2] \times[0,1]: f_{3}(c, x)<f_{4}(c, x)\right\} .
$$

Since $f_{2}(c, x) \geq 0$ in $[1,2] \times[0,1]$, so

$$
\sigma:=\frac{-c f_{2}(c, x)}{2\left(f_{3}(c, x)-f_{4}(c, x)\right)} \geq 0, \quad(c, x) \in \Omega_{2} .
$$

If $\sigma \geq 1$, i.e., if $c f_{2}(c, x)+2\left(f_{3}(c, x)-f_{4}(c, x)\right) \geq 0$, then

$$
\begin{aligned}
F_{1}(c, x, y) & \leq F_{1}(c, x, 1) \\
& =f_{1}(c, x)+c f_{2}(c, x)+f_{3}(c, x), \quad(c, x) \in \Omega_{2}, y \in[0,1] .
\end{aligned}
$$

and repeating the argumentation of Part (a) we get the inequality (2.14). 
If $\sigma<1$, i.e., if $c f_{2}(c, x)+2\left(f_{3}(c, x)-f_{4}(c, x)\right)<0$, then

$$
\begin{aligned}
F_{1}(c, x, y) & \leq F_{1}(c, x, \sigma)=\frac{-c^{2} f_{2}^{2}(c, x)}{4\left(f_{3}(c, x)-f_{4}(c, x)\right)}+f_{1}(c, x)+f_{4}(c, x) \\
& \leq \frac{\left[-2\left(f_{3}(c, x)-f_{4}(c, x)\right)\right]^{2}}{4\left(f_{3}(c, x)-f_{4}(c, x)\right)}+f_{1}(c, x)+f_{4}(c, x) \\
& \leq f_{1}(c, x)+f_{3}(c, x)+2 f_{4}(c, x)=: F_{2}(c, x), \quad(c, x) \in \Omega_{2} .
\end{aligned}
$$

Set $t:=c^{2}$. Clearly, $t \in[1,4]$. Define

$$
\tilde{F}_{2}(t, x):=(4-t) F_{2}(\sqrt{t}, x), \quad(\sqrt{t}, x) \in \Omega_{2} .
$$

A simple computing yields

$$
\begin{aligned}
\tilde{F}_{2}(t, x)= & (4-t)\left\{t x\left[3 t+(44-5 t) x+(40-t) x^{2}\right]+t(4-t) x^{4}\right. \\
& +32(4-t)+28 x^{2}(4-t)+36 t x\left(1-x^{2}\right)+4(4-t) x^{4} \\
& \left.+72[t+(4-t) x]\left(1-x^{2}\right)\right\} \\
= & -\left\{-64\left(8+18 x+7 x^{2}-18 x^{3}+x^{4}\right)\right. \\
& +16\left(-2+27 x+21 x^{2}-37 x^{3}+x^{4}\right) t \\
& +4\left(10-12 x-9 x^{2}+20 x^{3}+x^{4}\right) t^{2} \\
& \left.+x\left(3-5 x-x^{2}-x^{3}\right) t^{3}\right\}, \quad(\sqrt{t}, x) \in \Omega_{2}
\end{aligned}
$$

Hence and by Proposition 2.3 we have

$$
\frac{\partial}{\partial t} \tilde{F}_{2}(t, x)=-\Psi(t, x)<0, \quad(\sqrt{t}, x) \in \Omega_{2},
$$

where the function $\Psi$ is defined by (2.6). Thus the function $[1,4] \ni t \mapsto \tilde{F}_{2}(t, \cdot)$ is decreasing, and therefore we have

$$
\tilde{F}_{2}(t, x) \leq \tilde{F}_{2}(1, x)=9\left(56+85 x+17 x^{2}-71 x^{3}+5 x^{4}\right)<1024, \quad x \in[0,1]
$$

Indeed, the last inequality is true since, as easy to verify the inequality

$$
-520+765 x+153 x^{2}-639 x^{3}+45 x^{4}<0, \quad x \in[0,1]
$$

holds. Thus the inequality (2.17) confirms the inequality (2.14). 
II. Assume that $c \in(0,1)$. Then by (2.13) we have

$$
\begin{aligned}
F & (c, x, y) \\
& \leq f_{1}(c, x)+\frac{1}{c} f_{2}(c, x) y+f_{3}(c, x) y^{2}+f_{4}(c, x)\left(1-y^{2}\right) \\
& =f_{1}(c, x)+f_{4}(c, x)+\frac{1}{c} f_{2}(c, x) y+\left(f_{3}(c, x)-f_{4}(c, x)\right) y^{2} \\
& =: F_{3}(c, x, y) .
\end{aligned}
$$

(a) Consider the case $f_{3}(c, x) \geq f_{4}(c, x)$ in $(0,1) \times[0,1]$. Let

$$
\Omega_{3}:=\left\{(c, x) \in(0,1) \times[0,1]: f_{3}(c, x) \geq f_{4}(c, x)\right\} .
$$

By (2.18) we get

$$
\begin{aligned}
F_{3}(c, x, y) & \leq F_{3}(c, x, 1) \\
& =f_{1}(c, x)+\frac{1}{c} f_{2}(c, x)+f_{3}(c, x), \quad(c, x) \in \Omega_{3}, y \in[0,1] .
\end{aligned}
$$

Set $t:=4-c^{2}$. Clearly, $t \in(3,4)$. Define

$$
\tilde{F}_{3}(t, x):=t F_{3}(\sqrt{4-t}, x, 1), \quad(\sqrt{4-t}, x) \in \Omega_{3}
$$

A simple computing yields

$$
\begin{aligned}
\tilde{F}_{3}(t, x)= & t\left\{3(4-t)^{2} x+(4-t)(24+5 t) x^{2}+(4-t)(36+t) x^{3}+(4-t) t x^{4}\right. \\
& +4\left(1-x^{2}\right)\left[12-3 t+4(9-t) x+t x^{2}\right] \\
& \left.+32 t+28 t x^{2}+36(4-t) x\left(1-x^{2}\right)+4 t x^{4}\right\} \\
= & 48\left(1+7 x+x^{2}-3 x^{3}\right) t+4\left(5-19 x+10 x^{2}+5 x^{3}+x^{4}\right) t^{2} \\
& -x\left(-3+5 x+x^{2}+x^{3}\right) t^{3}, \quad(\sqrt{4-t}, x) \in \Omega_{3}
\end{aligned}
$$

Hence and by Proposition 2.4 we have

$$
\frac{\partial}{\partial t} \tilde{F}_{3}(t, x)=\Phi(t, x)>0, \quad(\sqrt{4-t}, x) \in \Omega_{3},
$$

where the function $\Phi$ is defined by (2.8). Thus the function $(3,4) \ni t \mapsto \tilde{F}_{1}(t, \cdot)$ is increasing, and therefore we have

$$
\tilde{F}_{3}(t, x) \leq 512+320 x+512 x^{2}-320 x^{3} \leq 1024
$$


Indeed, the last inequality is true since so is the following one

$$
-512+320 x+512 x^{2}-320 x^{3}=\left(1-x^{2}\right)(320 x-512) \leq 0, \quad x \in[0,1] .
$$

Thus the inequality (2.19) confirms the inequality (2.14).

(b) Consider the case $f_{3}(c, x)<f_{4}(c, x)$ in $(0,1) \times[0,1]$ which is equivalent to

$$
\begin{aligned}
32 & -8 c^{2}+28 x^{2}-7 c^{2} x^{2}+9 c^{2} x-9 c^{2} x^{3}+4 x^{4}-c^{2} x^{4} \\
& <9 c^{2}-9 c^{2} x^{2}+36 x-9 c^{2} x-36 x^{3}+9 c^{2} x^{3}
\end{aligned}
$$

for $c \in(0,1)$ and $x \in[0,1]$. Note that

$$
17-18 x-2 x^{2}+18 x^{3}+x^{4}>0, \quad x \in[0,1] .
$$

Thus the inequality (2.20) can be written as

$$
c^{2}>\frac{32-36 x+28 x^{2}+36 x^{3}+4 x^{4}}{17-18 x-2 x^{2}+18 x^{3}+x^{4}}, \quad c \in(0,1), x \in[0,1] .
$$

However,

$$
\frac{32-36 x+28 x^{2}+36 x^{3}+4 x^{4}}{17-18 x-2 x^{2}+18 x^{3}+x^{4}} \geq 1, \quad x \in[0,1] .
$$

Indeed, the above inequality is equivalent to

$$
32-36 x+28 x^{2}+36 x^{3}+4 x^{4} \geq 17-18 x-2 x^{2}+18 x^{3}+x^{4}, \quad x \in[0,1],
$$

which by simplifying is equivalent to the true inequality

$$
(x-1)^{4}+10 x^{3}+4(x-1)^{2}+6 x \geq 0, \quad x \in[0,1] .
$$

Thus by (2.21) and (2.22) it follows that $c \geq 1$ which contradicts the assumption.

III. At the end assume that $c=0$. Then by (2.13) we have

$$
\begin{aligned}
F(0, x, y) & =16\left(\left(8-9 x+7 x^{2}+9 x^{3}+x^{4}\right) y^{2}+9 x\left(1-x^{2}\right)\right) \\
& \leq 16\left(8+7 x^{2}+x^{4}\right) \leq 256, \quad x \in[0,1], y \in[0,1] .
\end{aligned}
$$

Summarizing, from all considering cases it follows that the inequality (2.14) holds which together with (2.12) shows (2.9).

Remark 2.6 Although the constant 8/9 improves essentially the estimates found in [1] and [19], it is not the best possible. To find the sharp estimate of the Hankel determinant $H_{3,1}(f)$ for starlike functions is still an open problem.

Acknowledgements We would like to express gratitude to the referees for their constructive comments and suggestions that helped to improve the clarity of this manuscript. 
Open Access This article is distributed under the terms of the Creative Commons Attribution 4.0 International License (http://creativecommons.org/licenses/by/4.0/), which permits unrestricted use, distribution, and reproduction in any medium, provided you give appropriate credit to the original author(s) and the source, provide a link to the Creative Commons license, and indicate if changes were made.

\section{References}

1. Babalola, K.O.: On $\mathrm{H}_{3}$ (1) Hankel determinants for some classes of univalent functions. In: Cho, Y.J. (ed.) Inequality Theory and Applications, vol. 6, pp. 1-7. Nova Science Publishers, New York (2010)

2. Bansal, D., Maharana, S., Prajapat, J.K.: Third order Hankel determinant for certain univalent functions. J. Korean Math. Soc. 52(6), 1139-1148 (2015)

3. Cho, N.E., Kowalczyk, B., Kwon, O.S., Lecko, A., Sim, Y.J.: The bounds of some determinants for starlike functions of order alpha. Bull. Malay. Math. Sci. Soc. 41, 523-535 (2018)

4. Cho, N.E., Kowalczyk, B., Kwon, O.S., Lecko, A., Sim, Y.J.: The bound of the Hankel detrminant for strongly starlike functions of order alpha. J. Math. Inequal. 11(2), 429-439 (2017)

5. Goodman, A.W.: Univalent Functions. Mariner, Tampa (1983)

6. Janteng, A., Halim, S.A., Darus, M.: Coefficient inequality for a function whose derivative has a positive real part. J. Inequal. Pure Appl. Math. 7(2), 1-5 (2006)

7. Kwon, O.S., Lecko, A., Sim, Y.J.: On the fourth coefficient of functions in the Carathéodory class. Comp. Meth. Funct. Theory 18, 307-314 (2018)

8. Lee, S.K., Ravichandran, V., Supramanian, S.: Bound for the second Hankel determinant of certain univalent functions. J. Inequal. Appl. 2013(281), 1-17 (2013)

9. Libera, R.J., Zlotkiewicz, E.J.: Early coefficients of the inverse of a regular convex function. Proc. Am. Math. Soc. 85(2), 225-230 (1982)

10. Libera, R.J., Zlotkiewicz, E.J.: Coefficient bounds for the inverse of a function with derivatives in $\mathcal{P}$. Proc. Am. Math. Soc. 87(2), 251-257 (1983)

11. Livingston, A.E.: The coefficients of multivalent close-to-convex functions. Proc. Am. Math. Soc. 21(3), 545-552 (1969)

12. Mishra, A.K., Gochhayat, P.: Second Hankel determinat for a class of analytic functions defined by fractional derivative. Int. J. Math. Math. Sci. Article ID 153280 (2008)

13. Pommerenke, C.: On the coefficients and Hankel determinant of univalent functions. J. Lond. Math. Soc. 41, 111-122 (1966)

14. Pommerenke, C.: Univalent Functions. Vandenhoeck \& Ruprecht, Göttingen (1975)

15. Prajapat, J.K., Bansal, D., Singh, A., Mishra, A.K.: Bounds on third Hankel determinant for close-toconvex functions. Acta Univ. Sapientae Mathematica 7(2), 210-219 (2015)

16. Raza, M., Malik, S.N.: Upper bound of the third Hankel determinant for a class of analytic functions related with lemniscate of Bernoulli. J. Inequal. Appl. 2013(412), 8 (2013)

17. Shanmugam, G., Stephen, B.A., Babalola, K.O.: Third Hankel determinant for $\alpha$-starlike functions. Gulf J. Math. 2(2), 107-113 (2014)

18. Sudharsan, T.V., Vijayalaksmi, S.P., Sthephen, B.A.: Third Hankel determinant for a subclass of analytic functions. Malaya J. Math. 2(4), 438-444 (2014)

19. Zaprawa, P.: Third Hankel determinants for subclasses of univalent functions. Mediter. J. Math. 14(1), 10 (2017) 